(C) 2015 IEEE. Personal use of this material is permitted. Permission from IEEE must be obtained for all other uses, in any current or future media, including reprinting/republishing this material for advertising or promotional purposes, creating new collective works, for resale or redistribution to servers or lists, or reuse of any copyrighted component of this work in other works. 


\title{
Classification of Migraine Stages based on Resting- State EEG Power
}

\author{
Ze-Hong Cao ${ }^{1,2}$, Li-Wei Ko ${ }^{2,3}$, Kuan-Lin Lai, ${ }^{4,5}$, Song-Bo Huang ${ }^{1,2}$, Shuu-Jiun Wang ${ }^{4,5^{*}}$, Chin-Teng Lin ${ }^{1,2^{*}}$, Fellow, IEEE \\ ${ }^{1}$ Institute of Electrical Control Engineering, National Chiao-Tung University, Hsinchu, Taiwan \\ ${ }^{2}$ Brain Research Center and I-RiCE Center, National Chiao-Tung University, Hsinchu, Taiwan \\ ${ }^{3}$ Institute of Bioinformatics and Systems Biology, National Chiao-Tung University, Hsinchu, Taiwan \\ ${ }^{4}$ Neurological Institute, Taipei Veterans General Hospital, Taipei, Taiwan \\ ${ }^{5}$ Department of Neurology, National Yang-Ming University, Taipei, Taiwan \\ *Email: ctlin@mail.nctu.edu.tw, sjwang@vghtpe.gov.tw
}

\begin{abstract}
Migraine is a chronic neurological disease characterized by recurrent moderate to severe headaches during a period like one month often in association with symptoms in human brain and autonomic nervous system. Normally, migraine symptoms can be categorized into four different stages: inter-ictal, pre-ictal, ictal, and post-ictal stages. Since migraine patients are difficult to know when they will suffer migraine attacks, therefore, early detecting attacks becomes an important issue, especially for low-frequency migraine patients who have less than 5 times attacks per month. The main goal of this study is to develop a migrainestage classification system based on migraineurs' resting-state EEG power. We collect migraineurs' 01 and 02 EEG activities during closing eyes from occipital lobe to identify pre-ictal and non-pre-ictal stages. Self-Constructing Neural Fuzzy Inference Network (SONFIN) is adopted as the classifier in the migraine stages classification which can reach the better classification accuracy $(66 \%)$ in comparison with other classifiers. The proposed system is helpful for migraineurs to obtain better treatment at the right time.
\end{abstract}

Keywords-Migraine; Pre-ictal; Resting State; EEG Power; Classification

\section{INTRODUCTION}

Migraine is a chronic neurological disease characterized by recurrent moderate to severe headaches during a period like one month often in association with symptoms in human brain and autonomic nervous system. It becomes one of the most common neurological diseases in the developed world $[1,2]$. Before headache attack suffering, migraine patients sometimes will have aura such as sensitivity to light and noise, nausea, vomiting, dizziness and eyesight changes [3, 4], but around 70 percent of migraine patients are in the type without aura [5]. Therefore, how to early detect migraine attack for the migraine without aura group becomes an important issue, especially in pre-clinical medicine.

Though the pathophysiology of migraine is still unclear, an altered state of brain excitability may be the fundamental path mechanism [6]. Migraine patients behave a kind of cortical fluctuating dysfunction compared with healthy people [7], and are observed to show an increase cortical activity with respect to healthy people [8]. It is considered that brain cortex power changes can serve as a neurophysiological biomarker for detecting migraine attacks.

In the past literatures, many EEG studies have addressed the brain excitability of migraine patients on different brain areas including somatosensory, visual and motor cortices producing somatosensory evoked potential (SEP) and visual evoked potential (VEP) [9], but very less studies are exploring the ways to detect different migraine stages without any external stimuli. In this study, we develop a migraine stage classification system based on the resting EEG power. There are four different migraine stages: interictal, pre-ictal, ictal and post-ictal. The pre-ictal and postictal stages are defined to be the periods of 36 hours before and after migraine attack, respectively. Attack period larger than 36 hours is defined as the inter-ictal stage. Since the pre-ictal stage is a critical timing for medical doctors to provide early treatment [10], we therefore focus on identifying pre-ictal and non-pre-ictal migraine stages in our classification system.

We analyze EEG data based on channel EEG power spectra, and use machine learning algorithms to distinguish pre-ictal stage and non-pre-ictal stages. We compared the prediction accuracy of different machine learning algorithm, such as Linear Bayes Normal Classifier (LDC), Quadratic Bayes Normal Classifier (QDC), N-Nearest Neighbor Classifier (KNN), Parzen Density Classifier (ParzenDC) and Support Vector Machine (SVM). Moreover, a fuzzy neural network called Self-Constructing Neural Fuzzy Inference Network (SONFIN) is applied to improve the classification accuracy in this study, which outperformed the compared learning algorithms in EEG studies [11]. The developed classification system can help to early detect migraine attacks and assists patients to take precaution of available therapies.

\section{MATERIALS AND METHODS}

\section{A. Participants}

One hundred and eight migraineurs (18 men and 90 women, mean age: 35.9 \pm 8.9 years), diagnosed by neurologists in Taipei Veterans General Hospital as low 
attack frequency patients (1-5 attacks per month), were invited as subjects in this study. All subjects were required to write headache diary to record recent migraine attacks. The diary would help us to know when the attacks started and ended. According to the second edition of the International Classification of Headache Disorders (ICDH-II) [10], individual migraine subject was classified into one of the migraine stages (inter-ictal, pre-ictal, ictal and post-ictal) based on their recorded headache diary [12] and symptom descriptions given by patient themselves (as show in Fig. 1). When patients had migraine attacks, they were considered to be in the ictal stage. The period of 36 hours before ictal stage is defined as pre-ictal stage. The 36 hours after ictal stage is defined as post-ictal stage. The period between pre-ictal stage and post-ictal stage is defined as inter-ictal stage. The Institutional Review Board of the Veterans General Hospital, Taipei, Taiwan, approved the study. Informed consent was obtained from all subjects before this study. The distribution of subjects' information in different groups is shown in Table I.

\section{B. Resting state EEG recording}

This experiment was performed at a static and lightless room in the Taipei Veterans General Hospital, and the environment setting is shown in the Figure 2. In the first 2 minutes, subjects were instructed to take several deep breathings to adapt to the environment. Then subjects were instructed to open their eyes for 30 seconds and close their eyes for 30 seconds three times in total (as show in Fig. 3), meanwhile EEG data were recorded at a sampling rate of $256 \mathrm{~Hz}$ by Nicolet One EEG system (natus Ltd., US). The EEG channels (FP1, FP2, F7, F3, Fz, F4, F8, T3, C3, Cz, C4, T4, T5, P3, Pz, P4, T6, O1, O2) were placed according to the international 10-20 system (as shown in Fig. 2). The Fz and $\mathrm{Pz}$ were used as the reference channels.

\section{EEG data analysis}

All of EEG data were analyzed by Matlab (The Mathworks, Inc.) with EEGLAB. EEGLAB is an open source Matlab toolbox for electrophysiological signal processing (http://sccn.ucsd.edu/eeglab/), which consists of artifact signal rejection, time/frequency analysis, and several visualized modes for EEG signal. Figure 4 shows the analytical procedure of EEG signal process and classification model building.

TABLE I.

DISTRIBUTION OF SUBJECT IN DIFFERENT GROUPS

\begin{tabular}{|c|c|c|c|c|}
\hline Stages & $\begin{array}{c}\text { Subject } \\
\text { Number }\end{array}$ & $\begin{array}{c}\text { Men } \\
\text { Number }\end{array}$ & $\begin{array}{c}\text { Women } \\
\text { Number }\end{array}$ & $\begin{array}{c}\text { Average } \\
\text { Age }\end{array}$ \\
\hline Inter-ictal & 74 & 9 & 65 & $34.5 \pm 8.8$ \\
\hline Pre-ictal & 13 & 6 & 7 & $37.0 \pm 9.1$ \\
\hline Ictal & 11 & 2 & 9 & $39.4 \pm 9.5$ \\
\hline Post-ictal & 10 & 2 & 9 & $38.2 \pm 11.1$ \\
\hline Total & 108 & 18 & 90 & $35.9 \pm 8.9$ \\
\hline
\end{tabular}

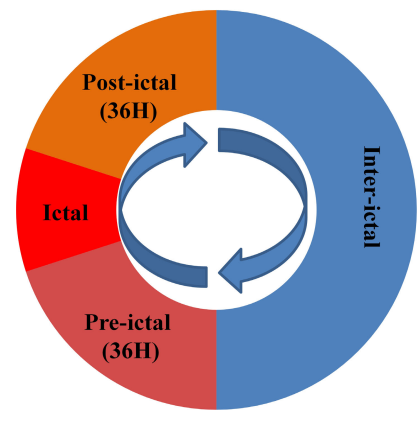

Fig. 1. Migraine cycle

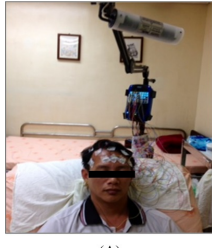

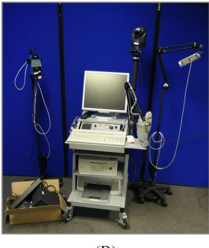

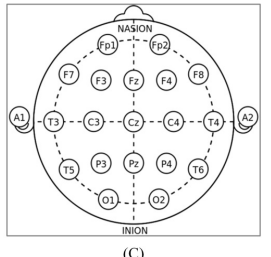

Fig. 2. Environmental setting. (A) Subjects who equipped Nicolet One system stayed in the lightless room. (B) Nicolet One System, EEG recording system, central control PC and EEG signal amplifier. (C) Channels are placed based on international 10-20 system.

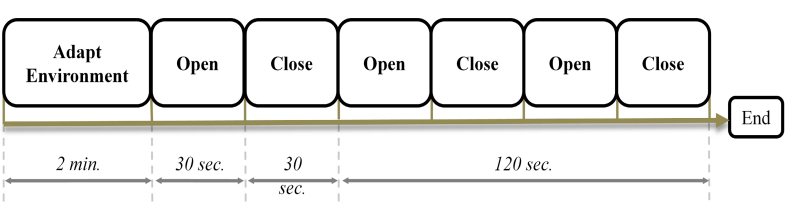

Fig. 3. The analytical procedure of EEG signal processing

\section{1) EEG Data Pre-Procesing}

The raw EEG signals were filtered through $1 \mathrm{~Hz}$ highpass and $50 \mathrm{~Hz}$ low-pass FIR filters. EEG activities were sampled at $256 \mathrm{~Hz}$ as the recording sampling rate. The filtered EEG data were then inspected automatically to identify and remove artifacts and noisy channels manually, such as detection of enormous outlier values that belong to one or more visible eye-blink or visible electromyography activity. The $\mathrm{O} 1$ and $\mathrm{O} 2$ channels were selected for EEG signals analysis and the three 30 -second closed-eye resting trials were extracted for the following analysis. Each 30second trial was separated into three 10-second epochs as shown in Fig. 5. Ten-second epochs were further transformed into the power spectra.

\section{2) EEG spectral features}

The time-domain resting-epochs EEG data were transformed into frequency domain for power calculation, feature selection and classifier learning. The used 256-point Fast Fourier Transform (FFT) window was fixed at 1-second long, 256-point data length, with 50\% overlapping with next window (see Fig. 5). The segment in each window was transformed into frequency domain respectively. When all the segments were transformed, we took average on these 
frequency domain transformations to represent the spectrum of the corresponding (resting) epoch.

\section{ClASSIFIERS DESIGN}

\section{A. Power Distribution in Differert Stages}

The pre-processing procedure on the collected EEG signals is shown in Fig. 4. After the processing, we calculated the EEG power in five different frequency bands (delta, theta, alpha, beta, and gamma). Figure 6 showed the

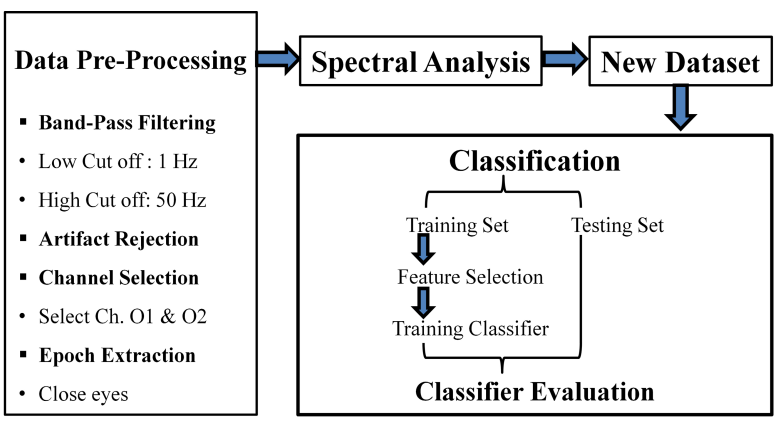

Fig. 4. System design flow for migraine states classification

Resting State with Closed Eyes

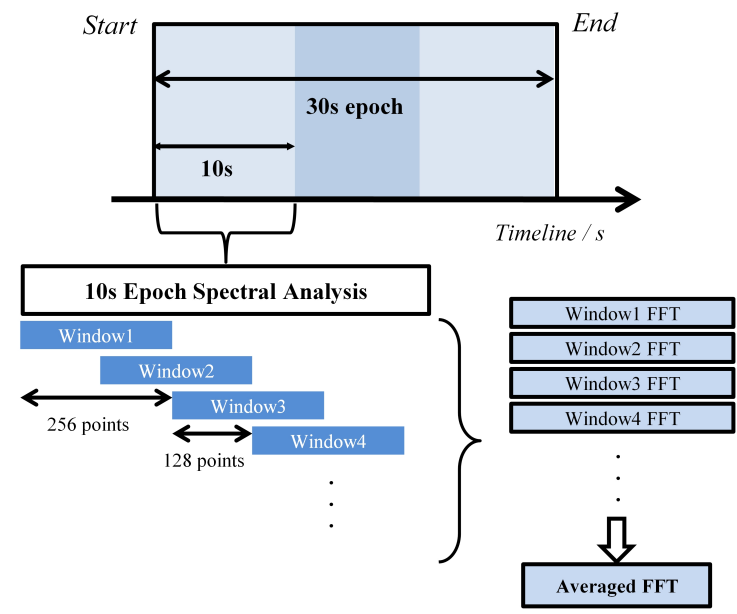

Fig. 5. EEG spectral analysis

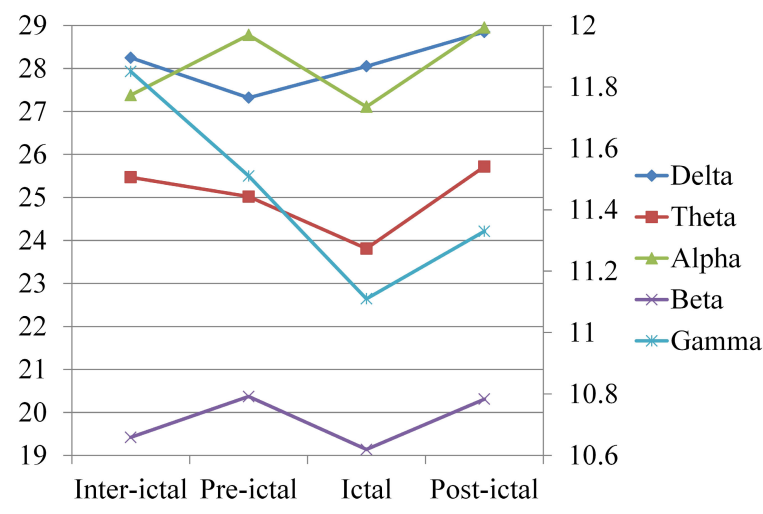

Fig. 6. Power distribution in resting state
EEG band powers in four different migraine stages: interictal, pre-ictal, ictal, and post-ictal stage. We can clearly see that the power spectrum of pre-ictal stage is different from that of the non-pre-ictal stage in delta, theta, alpha, beta, and gamma bands and thus these band powers can be used as biomarkers for identifying the pre-ictal and non-pre-ictal stages in a migraine cycle by a classifier.

\section{B. Classification Models}

The supervised learning approach was used to train the migraine-stage classifies. In preparing the training data for classifier learning, the forward feature selection method is adopted to evaluate a subset of features of the training data as a group for suitability. We selected the important feature sets pruning the data. Therefore, this forward feature selection method generated fewer, new features from the original data. Then, pairs of the determined feature sets and training data labels were fed into the machine learning algorithms to build classifiers. The learned classifiers are test by new sets of testing data through the same feature selection process.

Five popular classifiers were used and compared in this study: LDC, QDC, KNN, ParzenDC and SVM. The data set for each classifier is the same, which were filtered by 1 50 $\mathrm{Hz}$ band filter and generated by forward feature selection algorithm. After the model applies to classifiers, a 100 times Leave One-Out (LOO) cross- validation is used to evaluate the performance of each classifier.

Results of LOO cross-validation classification of the classifiers trained by different combinations of featureselection methods and learning algorithms are shown in Table II. The accuracies of all classifiers can reach about $50 \%$. The best combination is $\mathrm{KNN}$, which was used to selected features and SVM which was used to build a classifier, reaching the best accuracy of around 57\%.

\section{Improving Classification Model}

Self-constructing neural fuzzy inference network (SONFIN) is a general connectionist model of a fuzzy logic system [11]. It can construct an optimal structure and tune parameters automatically, and created dynamically as learning proceeds upon receiving on-line incoming training data. Both the structure and parameter identification schemes are done simultaneously during on-line learning, so the SONFIN can be used for normal operation at any time as learning proceeds without any assignment of fuzzy rules in advance. Moreover, the SONFIN can always find itself an economic network size, and the learning speed as well as the modeling ability are all appreciated. Using the SONFIN classifier with QDC feature selection, the classification accuracy is improved to about $66 \%$. 
TABLE II. CLASSIFICATION RESUlTS

\begin{tabular}{|c|c|c|c|c|c|c|c|}
\hline \multirow{6}{*}{ 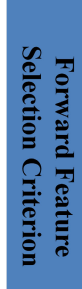 } & \multicolumn{7}{|c|}{ Classifier for Leave-One-Out Evaluation } \\
\hline & $\begin{array}{c}\text { Accuracy } \\
(\%)\end{array}$ & QDC & KNN & ParzenDC & LDC & SVM & SOFIN \\
\hline & QDC & $42.5 \pm 0.4$ & $43.5 \pm 0.6$ & $40.7 \pm 0.5$ & $38.9 \pm 0.5$ & $52.8 \pm 0.5$ & $66.3 \pm 0.5$ \\
\hline & KNN & $38.9 \pm 0.7$ & $57.4 \pm 0.7$ & $22.2 \pm 0.6$ & $40.7 \pm 0.4$ & $57.9 \pm 0.5$ & $60.2 \pm 0.6$ \\
\hline & ParzenDC & $38.9 \pm 0.5$ & $48.1 \pm 0.5$ & $39.8 \pm 0.5$ & $39.8 \pm 0.5$ & $55.1 \pm 0.6$ & $58.3 \pm 0.4$ \\
\hline & LDC & $33.3 \pm 0.6$ & $52.8 \pm 0.5$ & $39.8 \pm 0.6$ & $46.3 \pm 0.5$ & $50.3 \pm 0.7$ & $61.1 \pm 0.7$ \\
\hline & All Features & $41.2 \pm 0.4$ & $51.2 \pm 0.6$ & $39.3 \pm 0.5$ & $40.1 \pm 0.7$ & $57.1 \pm 0.7$ & $65.9 \pm 0.5$ \\
\hline
\end{tabular}

\section{DisCUSSION AND CONCLUSION}

Migraine headache attack (ictal stage) lasts 4 hours to 72 hours. Sometimes migraine patients cannot work properly since suffering headache attacks. The current treatment of migraine is medication or drug treatment. Studies have shown medication can effectively improve the condition in the period of prodromal symptoms. However, patients could not be in the exact timing of medication makes patients suffer because of the side effects of medication. Hence it is imperative and helpful to the migraine patients to predict the on-site of migraine attack.

In this study, we performed a fundamental study of migraine patients and realized an acceptable system that is capable of identifying migraine pre-ictal stage. In the proposed classifier design, the best combination is QDC method which was used to select proper features and SONFIN which was used to train the classifier. One key advantage of the proposed classification system is that the migraine-stage detection is based on resting EEG power without the need of any external tumulus to disturb patients' brain activities. Additionally, since only the occipital channels were used in our system, a simplified EEG recording system can be used in clinical applications conveniently and friendly. Overall, the proposed classification system is valuable and feasible to be applied in clinical applications. It can not only help migraine patients in their daily life, but also assist physicians to diagnose migraine patients by detecting the pre-ictal stage of the migraine patients and provide appropriate treatments in early stage before migraine attacks.

\section{ACKNOWLEDGMENTS}

This work was supported in part by the UST-UCSD International Center of Excellence in Advanced Bioengineering sponsored by the Taiwan National Science
Council I-RiCE Program under Grant Number: MOST-1032911-I-009-101, in part by the Aiming for the Top University Plan of National Chiao Tung University, the Ministry of Education, Taiwan, under Contracts 104W963, and in part by the National Science Council, Taiwan, under Contract MOST 103-2321-B-010-017. Research was also sponsored in part by the Army Research Laboratory and was accomplished under Cooperative Agreement Number W911NF-10-2-0022.

\section{REFERENCES}

B. K. Rasmussen, R. Jensen, M. Schroll, and J. Olesen, "Epidemiology of headache in a general population-a prevalence study," Journal of clinical epidemiology, vol. 44, pp. 1147-1157, 1991.

[2] R. B. Lipton, S. Diamond, M. Reed, M. L. Diamond, and W. F. Stewart, "Migraine diagnosis and treatment: results from the American Migraine Study II," Headache: The Journal of Head and Face Pain, vol. 41, pp. 638-645, 2001.

[3] R. De Simone, A. Ranieri, E. Marano, L. Beneduce, P. Ripa, L. Bilo, et al., "Migraine and epilepsy: clinical and pathophysiological relations," Neurological Sciences, vol. 28, pp. S150-S155, 2007.

[4] M. Bigal, R. Lipton, J. Cohen, and S. Silberstein, "Epilepsy and migraine," Epilepsy \& Behavior, vol. 4, pp. 13-24, 2003.

[5] R. W. Evans, "The clinical features of migraine with and without aura," Pract Neurol, vol. 13, pp. 26-32, 2014.

[6] S. K. Aurora and K. Welch, "Brain excitability in migraine: evidence from transcranial magnetic stimulation studies," Current opinion in neurology, vol. 11, pp. 205-209, 1998.

[7] M. Bjørk, L. Stovner, K. Hagen, and T. Sand, "What initiates a migraine attack? Conclusions from four longitudinal studies of quantitative EEG and steady-state visual-evoked potentials in migraineurs," Acta Neurologica Scandinavica, vol. 124, pp. 5663, 2011.

[8] P. Kropp and W.-D. Gerber, "Slow cortical potentials in migraine. Predictive value and possible novel therapeutic strategies to prevent an attack," Functional neurology, vol. 20, pp. 193-197, 2004.

[9] M. Valeriani, S. Rinalduzzi, and F. Vigevano, "Multilevel somatosensory system disinhibition in children with migraine," Pain, vol. 118, pp. 137-144, 2005.

[10] S. Silberstein, J. Olesen, M. G. Bousser, H. C. Diener, D. Dodick, M. First, et al., "The International Classification of Headache Disorders, (ICHD-II)-revision of criteria for 8.2 Medication-overuse headache," Cephalalgia, vol. 25, pp. 460465, 2005.

[11] C.-F. Juang and C.-T. Lin, "An online self-constructing neural fuzzy inference network and its applications," Fuzzy Systems, IEEE Transactions on, vol. 6, pp. 12-32, 1998.

[12] L.-W. Ko, K.-L. Lai, P.-H. Huang, C.-T. Lin, and S.-J. Wang, "Steady-state visual evoked potential based classification system for detecting migraine seizures," in Neural Engineering (NER), 2013 6th International IEEE/EMBS Conference on, 2013, pp. 1299-1302. 